\title{
Analytical Pyrolysis of Humic Substances: Pitfalls, Limitations, and Possible Solutions
}

\author{
C. Saiz-Jimenez \\ Instituto de Recursos Naturales y Agrobiologia, Consejo Superior de Investigaciones Cientificas, Apartado 1052 , \\ 41080 Sevilla, Spain
}

Analytical pyrolysis is a technique currently used for investigating structural features of complex macromolecular materials. However, pitfalls appear to be widespread in pyrolysis studies of humic substances, and serious limitations can be experienced during the analytical procedure due to thermal reactions and configuration of the pyrolysis units and chromatographic systems. Pyrolysis presents inconsistencies for chemical studies, and the interpretation of the chemical structure of humic substances in light of information provided by pyrolysis data is, to say the least, hazardous. This paper examines possible solutions to some of the problems encountered in pyrolysis.

\section{Introduction}

The chemical nature of humic substances has been a challenge to the ingenuity of scientists for more than 200 years, and in spite of the application of almost all available analytical instrumentation over the last four decades, knowledge of their nature and composition is still limited.

New insights into the structure of humic substances have inevitably come with the application of new analytical techniques. Many of the degradative methods so far employed are of limited value as the reaction products only partially reflect the structures of the building blocks and not much of their linkages. The interpretations could possibly be incorrect, as in many instances the naturally occurring units may have been altered before or after their release from the macromolecular structure $(1,2)$.

In a search for reliable methods capable of solving the intriguing aspects of humus chemistry, during the late seventies attention was focused on analytical pyrolysis, which has been applied to a variety of plant materials and soil organic matter fractions for the last 15 years $(3-5)$.

\section{Analytical Pyrolysis}

Analytical pyrolysis is considered to be a small-scale thermal degradation method that is very useful for the chemical characterization of materials from their pyrolysis products. This technique usually involves an integrated pyrolysis-analysis system that is carefully controlled to give reproducible results and that uses small (nanogram to microgram range) amounts of samples (6).

Analytical pyrolysis is not the ideal technique for investigating structural features of complex macromolecular materials, as thermal degradation of building blocks often results. However, it is probably the best technique employed so far for this purpose. This is due to its facility in investigating macromolecular materials in terms of pyrolysis products. Macromolecular materials are usually recalcitrant to any direct analytical approach, unless chemical degradation can be accomplished, to yield more affordable, lower molecular weight products. No chemical degradation, fractionation, purification, and time-consuming derivatization of reaction products are necessary for pyrolysis studies, which facilitates the analysis.

Considerable efforts have been made over the last decade to understand the chemical structure of humic substances in terms of evolved pyrolysis products. Of the various pyrolysis approaches, the most reliable seems to be pyrolysis-gas chromatography-mass spectrometry, as pyrolysis products can be separated by this method in the column of a gas chromatograph and identified by mass spectrometry. A comprehensive study of different soil humic fractions was reported by Saiz-Jimenez and de Leeuw (4) in which up to 322 compounds were identified in the pyrolysates. More specific studies on soil polysaccharides, fulvic acids, and polymaleic acid were carried out to establish structural relationships between them (7). Detailed studies on the most resistant part of a humic acid (hydrolyzed or persulfate oxidized residues) revealed 
that aliphatic biopolymers, similar to those encountered in plant cuticles, could form part of this humic moiety (8).

Most of the major classes of compounds and biomacromolecules found in humic substances were also apparent in pyrolysis studies (as their primary or secondary pyrolysis products), which indicates that pyrolysis is able to provide general information on complex mixtures of compounds or materials (8-11).

In a previous review (12), an attempt was made to summarize the most important milestones in pyrolysis studies of humic substances and, at the same time, to demonstrate how existing theories on the chemical structure of humic substances, from the sixties to the eighties, have decisively influenced structural data derived from pyrolysis, reducing or otherwise changing their impact. Even nowadays, pyrolysis data on humic substances are not correctly interpreted, and misunderstandings often occur in pyrolysis papers.

\section{Pitfalls in Analytical Pyrolysis}

Analytical pyrolysis can cause drastic modification of the original building blocks which may lead to incorrect conclusions on the structure. In fact, the most biased tendency has been to consider pyrolysis products as building blocks of the macromolecule.

In recent years, it has been stated that analytical pyrolysis can provide important clues for understanding the chemical structure of complex macromolecules (13). However, it appears that there is a need to scrutinize the information generated by this technique regarding humic substances as, in some cases, misleading inferences have been made. This can be exemplified in the pyrolysis of some biomacromolecules such as polysaccharides, proteins, lignins, etc.

Pyrolysis of plant and soil polysaccharides $(4,7,10)$ results in complex pyrolysates containing a wide variety of volatile, relatively low molecular weight compounds and some other compounds, mostly anhydrosugars. There is some agreement that thermal decomposition of polysaccharides (e.g., cellulose) is the result of two competing reactions: a dehydration to yield anhydrocellulose and a depolymerization of cellulose to yield primarily levoglucosan and minor anhydrosugar components. The production of levoglucosan and other anhydrosugars is the first step in the formation of volatiles from the pyrolysis of polysaccharides, and it appears that this reaction is the main process at high temperatures and high heating rates. The identification of aldehydes and ketones of different chain length, acids, furans, pyranones, anhydrosugars, and phenols (which include phenol, cresol, benzenediol, hydroxybenzaldehyde, and dihydroxyacetophenone) in the pyrolysate of cellulose (10) indicates that considerable thermal degradation and secondary reactions have been produced in the original building blocks.

Pyrolysis of proteins also produces secondary reactions as encountered in polysaccharides. In this case, the range of pyrolysis products is increased as the variety of building units or amino acids is also increased. As opposed to polysaccharide studies, protein or peptide pyrolysis shows a high number of unknown compounds, some of which have recently been identified $(11,14,15)$.

Alkylated pyrrolediones and pyrrolidinediones have been found in pyrolysates of proteins (11). These are cyclization products of the aliphatic amino acids alanine,

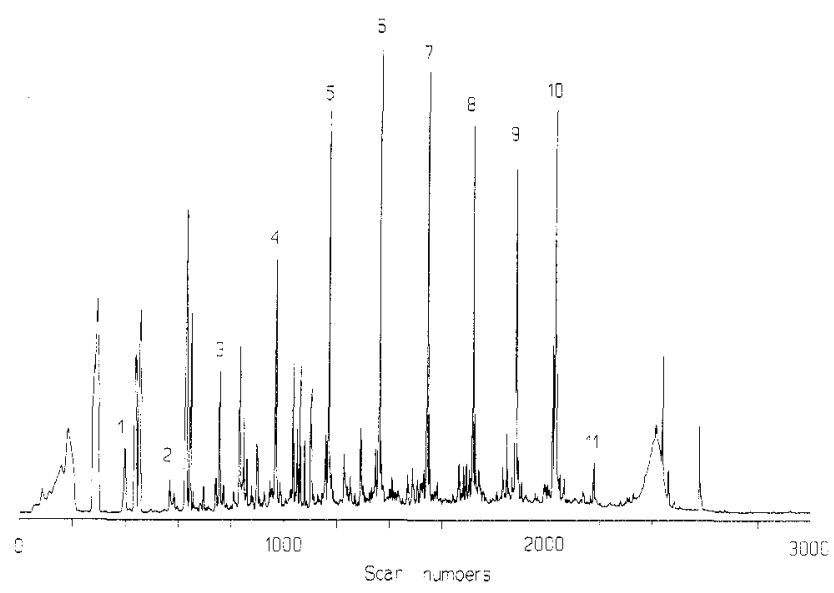

Flgure 1. Total lon current chromatogram of sodium oleate in the presence of elemental sulfur. Curle temperature, $590^{\circ} \mathrm{C}$. Peak numbers refer to the carbon number in the aliphatic chain of alkylbenzenes.

leucine, isoleucine, and valine when they appear combined in groups of two in the proteins. Therefore, in pyrolysis of proteins, secondary reactions are also produced which distance the chemical structure of pyrolysis products from that of their original amino acid constituents.

Lignin is perhaps one of the most appropriate biomacromolecules to study by pyrolysis. Several papers investigated its pyrolysis products in detail together with their significance as biomarkers $(16,17)$. Briefly, pyrolysis of lignins yields a variety of products derived from p-coumaryl, coniferyl, or syringyl alcohol units, depending on the type of lignin. Although these precursor phenols can be found in the pyrolysate, thus representing primary pyrolysis products, many other compounds represent intermediate steps in thermal degradation and probably secondary reaction products. Therefore, from the most simple compounds (such as phenol, guaiacol, and 2,6dimethoxyphenol, in which the propenyl side chain was split off) to the most complex precursor units (the abovementioned alcohols) a range of compounds with thermally modified functional groups can be readily assigned to lignin phenols.

Recently, it was found that analytical pyrolysis of fatty acids produced alkylated benzenes and naphthalenes. In fact, Saiz-Jimenez (18) proved that pyrolysis of unsaturated fatty acids and/or triglycerides in the presence of sulfur derivatives yielded series of alkylbenzenes, alkylthiophenes, alkylfurans, alkanes, alkenes, and alkanethiols. Moreover, the pyrolysis of a single compound-sodium oleate-in the presence of elemental sulfur yielded a complex pyrolysate in which the major compounds were the series of alkylbenzenes followed by the series of alkylthiophenes, as shown in Figure 1. Saiz-Jimenez (19) stated that unsaturated fatty acids and sulfur are commonly found in humic substances. Upon pyrolysis, fatty acids in the presence of inorganic sulfur or sulfurcontaining lignins and polysaccharides undergo decarboxylation, cyclization, and aromatization reactions, yielding homologous series of alkylbenzenes, alkythiophenes (incorporation of sulfur), or alkyfurans (incorporation of oxygen). The formation of homologous series of alkylated benzenes and naphthalenes from aliphatic precursors is of interest regarding the structural significance of these pyrolysis products in humic substances.

It is demonstrated that in general the structures of pyrolysis products from polysaccharides, proteins, lignins, 
and lipids are very different from those of parent units, which clearly illustrates the complexity of pyrolysates obtained from macromolecules with many different units. Therefore, pyrolysis presents inconsistencies for chemical studies; interpretation of the chemical nature of complex biomacromolecules in general, and humic substances in particular, in light of the information provided by pyrolysis data is, to say the least, hazardous.

Another common pitfall is to consider all compounds evolved from pyrolysis as pyrolysis products. Three possible origins can be traced for the majority of compounds identified in humic substances pyrolysates:

Evaporation. Free compounds present in the humic molecule evaporate quickly under pyrolysis (e.g., alkanes, fatty acids, etc.).

Pyrolysis. Structural units in a macromolecule split off under fast heating in an inert atmosphere, and lower molecular weight fragments evolve.

Combustion. Structural units in a macromolecule split off through burning in the presence of oxygen and are then incorporated into soils and humic substances.

In fact, lipids (including alkanes, fatty acids, dicarboxylic acids, ketones, etc.) are synthesized by microorganisms and plants and can be found as free, solvent-extractable compounds in soils and soil humic fractions (20-22). Therefore, the majority of them probably represents evaporation products when found in pyrolysates. Lipids are also originated upon combustion of fossil fuel and biomass and can, in certain soils, be the reflection of an input of airborne pollutants to the environment.

Alkylfurans are common pyrolysis and combustion products of carbohydrates and polysaccharides $(7,10,23)$, although they are also produced upon pyrolysis of fatty acids $(18,19)$. Rearrangements and thermal transformation of original glucopyranose units leading to most simple furans depend on the pyrolysis or combustion temperature, and these compounds can be found in charred plant materials or their condensates (23). Similarly, methoxylated phenols have been considered to be tracers from wood materials in the combustion of biomass (24) and the pyrolysis of wood and isolated lignins (17). Both types of compound - furans and methoxylated phenols - are widely distributed in soils due to agricultural practices (e.g., debris burning) or forest fires, and therefore it is sometimes difficult to discern an origin for the evaporation/pyrolysis products of the humic acids from these soils (23). In forest fires, the combustion products are incorporated into soils and humic substances and, subsequently, can evolve through evaporation in pyrolysis studies of the whole soil and/or their humic fractions.

Alkylated benzenes, naphthalenes, fluorenes, and phenanthrenes are produced upon the combustion of biomass or fossil fuels. They are widespread and usually represent pollutants introduced into the environment. Thus, evaporation/pyrolysis of environmental samples (charred brush, diesel soot, etc.) provides a wide variety of these alkyl aromatic compounds. Pyrolysis of plant stems also yields minor amounts of alkyl aromatics, demonstrating that these compounds are related to thermal degradation, as no long-chain alkylbenzenes have been reported in plant biomass (23). The input of alkyl aromatics and polycyclic aromatic hydrocarbons to the soil would lead to fast and complete binding by humic fractions, as reported by Schlautman and Morgan (25).
At present, it is impossible to distinguish between evaporation and pyrolysis products through the pyrolysis of humic substances, unless solvent extraction and/or lowtemperature desorption (e.g., $358^{\circ} \mathrm{C}$ ) have previously been accomplished $(8,20)$.

\section{Limitations in Analytical Pyrolysis}

One of the most intriguing facts in humus chemistry is the finding of considerable carboxyl-containing aromatic structures in fulvic and humic acids upon chemical oxidations (27) and in fulvic acids by column fractionation (28), which were not apparent in other degradative methods, including conventional pyrolysis. The presence of carboxyl groups was, however, evidenced by NMR (29), and therefore, data obtained from analytical pyrolysis do not conform with NMR data as far as functional groups are concerned.

In pyrolysates of fulvic and humic acids, no carboxylic groups are found other than those of a few fatty acids (mainly the $\mathrm{C}_{16}$ and $\mathrm{C}_{18}$ members) and rarely benzoic and vanillic acid $(4,7,30)$. The fatty acids are mostly believed to be evaporation (and not pyrolysis) products, as they can be extracted by organic solvents or chromatographically resolved at low temperature pyrolysis $(8,20,22)$. These fatty acids cannot account for the high carboxylic carbon resonances observed in NMR studies. In a few cases, these acids were identified in the pyrolysate as methyl esters, attributed to methylation produced by the methanol used to suspend and to apply the sample on the wire (7).

It was suspected that conventional chromatographic conditions do not evidence the carboxyl-containing pyrolysis products. This was proved in a previous paper (31), where solvent extraction vs analytical pyrolysis was applied to environmental samples. In fact, a complex mixture, in which fatty and dicarboxylic acids were the most abundant compounds, was resolved by solvent extraction, subsequent methylation, and GC/MS analysis. However, upon pyrolysis, a completely different pattern was obtained for the same samples, as the series of alkanes and alkenes predominated. It was suggested that carboxylic acids decarboxylate upon pyrolysis, yielding the corresponding alkanes and alkenes, so that structural units containing carboxyl groups are missing.

Another limitation is the pyrolysis temperature. Pyrolysis behavior of humic substances is highly dependent on the temperature selected. A soil humic acid subjected to different temperatures $\left(358,510,610,770^{\circ} \mathrm{C}\right)$ yields essentially distinct classes of evaporation and/or pyrolysis products $(4,8)$. In general, temperatures of $358^{\circ} \mathrm{C}$ originate evaporation of adsorbed phenols, dialkyl phthalates, and lipids (alkanes, fatty acids, etc.); pyrolysis of carbohydrates and polysaccharides; and some pyrolysis of lignin. Evaporation was demonstrated, as the same alkane and fatty acid distributions were obtained from humic acids after solvent extraction or thermal desorption at $358^{\circ} \mathrm{C}(8)$. Pyrolysis at $510^{\circ} \mathrm{C}$ is a good compromise for polysaccharide- and lignin-rich humic materials, as a well-balanced distribution of pyrolysis products from both biomacromolecules is obtained. Basically, fulvic acids respond better to temperatures around $500^{\circ} \mathrm{C}$. Pyrolysis at $610^{\circ} \mathrm{C}$ was also used for humic acid studies. Representative chromatograms were obtained for hydrolyzed humic acids provided that carbohydrate and protein 
components were released from the humic matrix, because to some extent they invade the chromatogram and mask other possible components. The use of higher temperatures is justified when a more in-depth insight is required from purified humic fractions containing refractory materials. Pyrolysis at $770^{\circ} \mathrm{C}$ has been used when studying resistant macromolecules. Residual humic acids, after acid hydrolysis and/or persulfate oxidation, react well at this high pyrolysis temperature, and an aliphatic biopolymer was suggested to be the main constituent of this resistant moiety (8). Hatcher and Clifford (32) confirmed these data in the pyrolysis at $300,500,600$ and $700{ }^{\circ} \mathrm{C}$ of Armadale humic acid. In light of these data, the choice of a temperature between 500 and $600{ }^{\circ} \mathrm{C}$, depending on the nature of the sample, appears appropriate for pyrolysis studies.

The weakest point in pyrolysis is, however, the transfer of data from pyrolysis to the whole macromolecule when the establishment of a chemical structure is intended. This is due to the following drawbacks: (a) Extensive thermal degradation of building blocks through secondary reactions, as exemplified in the pyrolysis of polysaccharides and proteins. (b) Compared to the tar, which is condensed onto the tube wall, and the carbonaceous residue, which remains in the pyrolysis chamber, a relatively low amount of volatile compounds can escape from the pyrolysis unit to the gas chromatograph. (c) Serious limitations in the analytical procedure are experienced due to restrictions in the chromatographic system (peak tailing, column polarity, oven temperature limit depending on the phase employed, etc.). Because of this, it is necessary to approach the subject cautiously, bearing in mind the real value of the pyrolysis products and to what extent they are representative of or can be related to structural units.

\section{Possible Solutions in Analytical Pyrolysis}

In an attempt to overcome some of these and other problems, attention was recently drawn to the method proposed by Challinor (33), which consists of simultaneous pyrolysis and derivatization with tetramethylammonium hydroxide (TMAH). Pyrolysis/methylation appeared to result in hydrolysis and methylation of the polar components, giving methyl esters of the polybasic acid, longchain fatty acids, and polyhydric alcohols, the original components of the resin studied.

Mulder et al. (34) applied the method to the study of phenolic acids, whether free or in complex biological matrices. While pyrolysis resulted in the decarboxylation of the acid in p-coumaric acid, pyrolysis/methylation prevented decarboxylation by protecting carboxyl groups.

De Leeuw and Baas (35) stated that in pyrolysis/ methylation, one has to distinguish between pyrolysis products sensu stricto and products resulting from bond breaking induced by chemical reagents at elevated temperatures. De Leeuw and Baas' opinion is that most of the products obtained can be explained by assuming a hydrolysis reaction followed by quantitative methylation of the fatty acid moieties.

Anderson and Winans (36) analyzed natural resins and resinites based on diterpenoid carboxylic acids. They were able to demonstrate that pyrolysis/methylation provided an accurate indication of the composition of these materials, with the exception of compounds having an additional hydroxyl and/or carbonyl group. The optimum pyrolysis temperature was found to be $480^{\circ} \mathrm{C}$.
The applicability of pyrolysis/methylation seems to be influenced by the pyrolysis temperature, a factor not stressed by previous authors. Ohtani et al. (37) suggested that at $400^{\circ} \mathrm{C}$ TMAH not only alkylates volatile decomposition products of aromatic polyesters but may also react directly with the polymer, yielding methyl derivatives of its constituents. However, at temperatures above $500^{\circ} \mathrm{C}$, decarboxylation occurred while at $650^{\circ} \mathrm{C}$ methylation was not complete, probably because chain scission is too rapid for completion of the reaction. It was concluded that 400 ${ }^{\circ} \mathrm{C}$ was the optimum pyrolysis temperature for aromatic polyesters.

In light of the preceding data, pyrolysis/methylation seems a candidate technique of potential interest for the study of the structure of humic substances.

\section{Pyrolysis/Methylation of Humic Substances and Related Materials}

In a first attempt to apply pyrolysis/methylation to the chemical characterization of humic fractions, Saiz-Jimenez et al. (38) investigated an aquatic fulvic acid and compared the data with those obtained by conventional pyrolysis. Similar classes and ranges of compounds were obtained in both cases. However, while in conventional pyrolysis, free fatty acids in the range of $\mathrm{C}_{10}-\mathrm{C}_{18}$ were identified; in pyrolysis/methylation, the range was $\mathrm{C}_{8}-\mathrm{C}_{18}$ but as their methyl ester. The most significant fact was the identification of furancarboxylic acids, benzenecarboxylic acids, and aliphatic dicarboxylic acids as their respective methyl esters in the methylated pyrolysate.

In this aquatic fulvic acid, a characteristic signature was the abundance of lignin-derived phenols, among which the $p$-coumaryl, guaiacyl, and syringyl series were distinguished. The identification of derivatives from the three lignin units agrees with the nature of the lignin synthesized by the autochthonous vegetation, grasses being the dominant plants. The presence of 3,4,5-trimethoxybenzoic acid and benzenecarboxylic acids was of interest as they represent final steps in the oxidation of side chains in lignin units through microbial degradation. Benzenecarboxylic acids and their methyl and methoxy derivatives have been identified in terrestrial fulvic acids by using nondestructive methods (28), and their presence in the aquatic fulvic acid is not surprising as this fulvic acid originates mostly from runoff of soil materials after a heavy rainfall period (39).

When pyrolysis/methylation was applied to fulvic acids from a meadow soil (Typic Xerochrept) and a podzol soil (Haplaquod), extended series of methyl esters of fatty acids (from $\mathrm{C}_{4}$ to $\mathrm{C}_{30}$ ), dicarboxylic acids $\left(\mathrm{C}_{6}-\mathrm{C}_{26}\right)$, and benzoic, benzenedicarboxylic, and benzenetricarboxylic acids were obtained. It is worth noting that phenolic and benzenecarboxylic acids constituted a significant part of the aromatic pyrolysis products in podzol fulvic acid; however, a low number of lignin phenols were identified. The reverse was true for the meadow fulvic acid. It was speculated that lignins have to some extent completed their microbial oxidation before accumulating in the $\mathrm{B}_{\mathrm{h}}$ horizon of the podzol soil, while the fulvic acid isolated from the A horizon of a meadow soil contains lignin units in different decomposition stages, from which the basic $\mathrm{C}_{6}-\mathrm{C}_{3}$ unit can still be traced (39).

Saiz-Jimenez et al. (38) stated that the finding of benzenecarboxylic acids would represent structural con- 

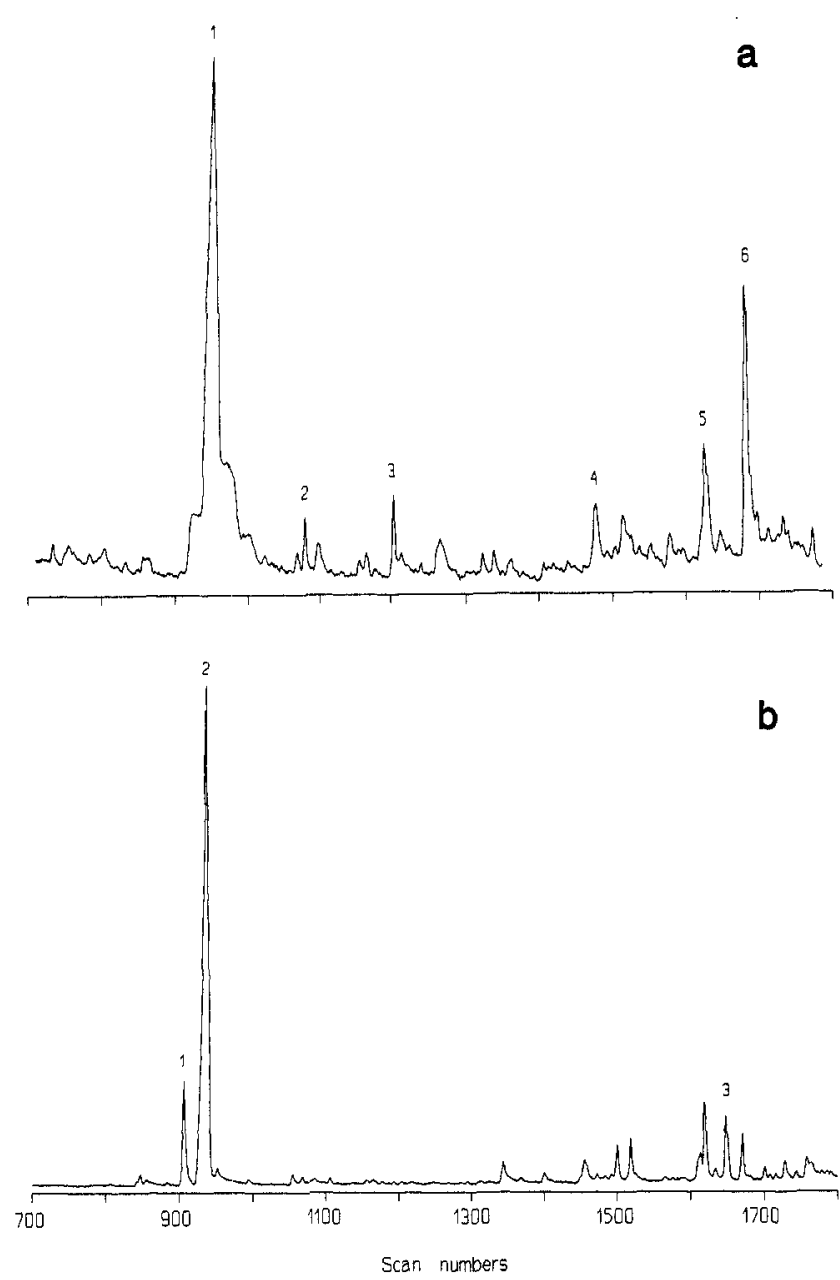

Figure 2. Partial total ion current chromatograms of polymaleic acid. (a) Conventional pyrolysis. Curie temperature, $500^{\circ} \mathrm{C}$. Major compounds are 3,4-dimethyl-2,5-furandione (1), 3-ethyl-4-methyl-2,5-furandione (2), 3,4-diethyl-2,5-furandione (3), phthallc anhydride (4), methyl-1,3isobenzofurandione (5), and 2-carboxybenzeneacetic acid (6). (b) Pyrolysis/methylation. Curle temperature, $300^{\circ} \mathrm{C}$. Major compounds are butenediolc acid dimethyl ester (1), butanedioic acid dimethyl ester (2), and cyclohexanedicarboxylic acid dimethyl ester, tentatively (3).

stituents of fulvic acids not previously identified in pyrolysis. However, a more extensive characterization of pyrolysis products from fulvic acids is needed for a complete demonstration of the origin of benzenecarboxylic acid in pyrolysis/methylation. In order to shed further light on the mechanisms of pyrolytic methylation, an aliphatic polycarboxylic acid, polymaleic acid, was selected. Polymaleic acid is formed by the hydrolysis of basecatalyzed homopolymerized maleic anhydride (40), resulting in a water-soluble brown polymer that has been considered chemically and structurally related to soil fulvic acids (41).

In previous studies, Bracewell et al. (42) indicated that, on pyrolysis, polymaleic acid undergoes fragmentation with cyclization, elimination, and decarboxylation reactions and yields two characteristic products, 2-cyclopenten-1-one and 3,4-dimethyl-2,5-furandione. However, Saiz-Jimenez and de Leeuw (7) found 2,5-furandione and 3,4-dimethyl-2,5furandione as major products. Monomethyl esters of butenedioic and butanedioic acids were also important.

Figure 2 shows the TIC chromatograms obtained from polymaleic acid pyrolysates using conventional $\left(500^{\circ} \mathrm{C}\right)$ and methylation $\left(300^{\circ} \mathrm{C}\right)$ pyrolyses. Analytical conditions were previously reported (39). Figure 2 clearly illustrates the different data obtained when both methods are applied to the same sample, indicating the importance of the protection of functional groups. Also, comparison of both techniques revealed that cyclization reactions (e.g., 3,4dimethyl-2,5-furandione) are produced under conventional pyrolysis in aliphatic precursors containing two carboxyl groups, whereas pyrolysis/methylation prevents this process by protecting the carboxyl groups.

The data herein presented, together with those obtained previously (7), shed some light on the pyrolysis mechanism. In fact, the major compound in pyrolysis/methylation was butanedioic acid dimethyl ester (which could be the main structural unit in the polymer) together with a lesser amount of butenedioic acid dimethyl ester; in conventional pyrolysis using methanol to dissolve the sample and deposit it on the wire, the monomethyl esters of butenedioic and butanedioic acids were relatively abundant due to partial methylation before pyrolysis. However, in conventional pyrolysis with solid samples (see Figure 2a), the butenedioic and butanedioic acids were absent due to cyclization reactions. This could be interpreted that pyrolysis of polymaleic acid originates fragmentation of the backbone in one building block, butanedioic acid, not identified as such in conventional pyrolysis, but resolvable as mono- or dimethyl esters when polymaleic acid is partially or fully methylated.

A matter that also merits discussion is the comparison between pyrolysis/methylation at 300 and $500^{\circ} \mathrm{C}$ (not shown here). From studying the products evolved at both pyrolysis temperatures, it becomes obvious that the higher the temperature, the higher the number of pyrolysis products generated. In this case, a few minor aromatic compounds were identified at $500^{\circ} \mathrm{C}$, particularly methyl esters of dihydroxybenzenepropanoic acid and methylbenzenedicarboxylic acid. This suggests either that pyrolysis/methylation of aliphatic polycarboxylic chains at relatively high temperatures is able to produce cyclization and aromatization of aliphatic structures or (more likely) that such aromatic structures were already formed during the maleic anhydride reactions, but only thermally evidenced at temperatures higher than $300^{\circ} \mathrm{C}$.

Benzenecarboxylic acids represent original components and were isolated from soil humic fractions using nondegradative methods (28), and the identification of their origin in humic substances is therefore of the utmost importance in order to rule out the possibility that these compounds could be produced under pyrolysis of certain types of aliphatic structures.

To gain more knowledge and prove the usefulness of pyrolysis/methylation for more complex materials, an agricultural soil humic acid from Russia (43) was investigated. This gives some clues on the behavior of humic acids in conventional pyrolysis and pyrolysis/methylation. Figure 3 shows the TIC chromatograms of the humic acid using these two procedures. There are striking differences between the two chromatograms. The predominating compounds in conventional pyrolysis were nitrogen derivatives, which are pyrolysis products of amino acids/ proteins $(14,15)$, lignin phenols $(16,17)$; 5-methyl-2furaldehyde, a pyrolysis product of carbohydrates/ polysaccharides $(4,10)$; and $n$-hexadecanoic acid, an evaporation product (8). Alkanes and alkylbenzenes were minor compounds. The identification of a few chlorinated compounds is highly suggestive of a chlorination process during the fractionation and purification procedures, as 


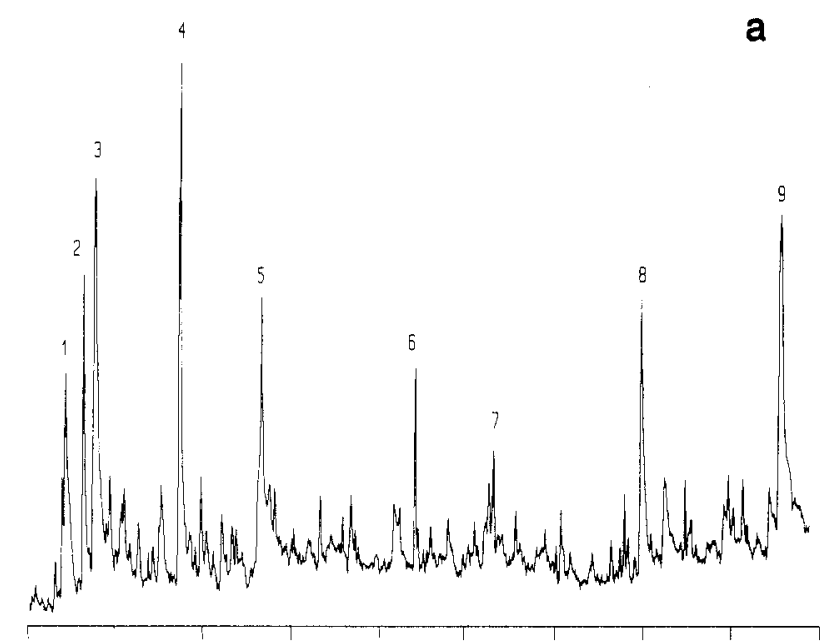

b

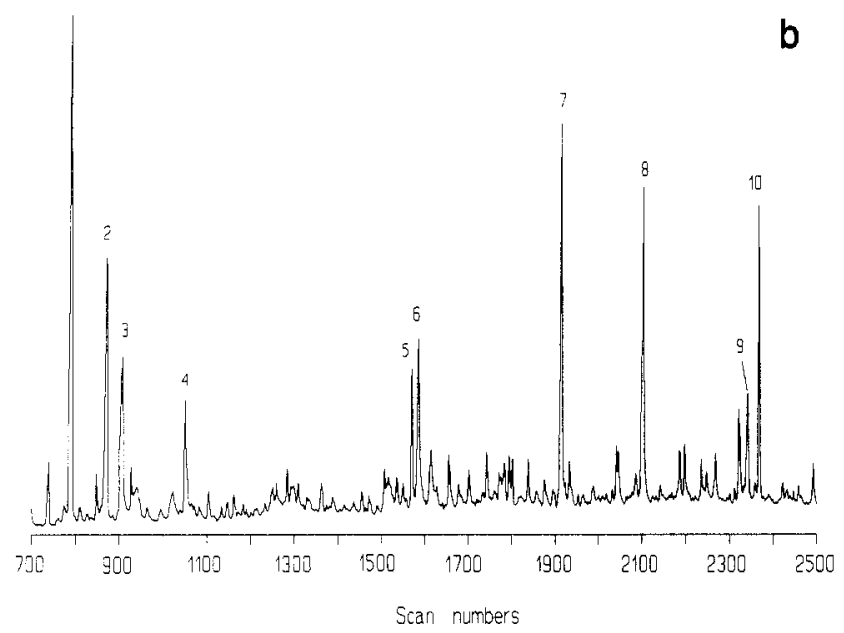

Figure 3. Partial total ion current chromatograms of a soll humic acid. (a) Conventional pyrolysis. Curle temperature, $500^{\circ} \mathrm{C}$. Major peaks are 5-methyl-2-furaldehyde (1), benzonitrile (2), phenol + benzofuran (3), gualacol (4), naphthalene (5), vinyinaphthalene (6), n-dodecane (7), diketodipyrrole (8), and $n$-hexadecanoic acid + dibutylphthalate (9). (b) Pyrolysis/methylation. Curie temperature, $500^{\circ} \mathrm{C}$. Major compounds are phosphoric acid trimethyl ester (1), N,N-dimethyloctanamide (2), 2-butenedioic acid dimethyl ester (3), benzoic acid methyl ester (4), methoxybenzoic acid methyl ester (5), 1,3,5-trimethyl-1,3,5-triazine2,4,6-(1H,3H,5H)trione (6), 3,4-dimethoxybenzoic acid methyl ester (7), 3,4,5-trimethoxybenzoic acid methyl ester (8), n-hexadecenoic acid methyl ester (9), and $n$-hexadecanolc acid methyl ester (10).

trichloroacetic acid was used. In contrast, pyrolysis/ methylation yielded methylated vanillic and syringic acids as major compounds, which were absent in conventional pyrolysis. Other important compounds were methylated phosphoric and benzoic acids, $N, N$-dimethyloctanamide, and trimethyltriazinetrione. While phosphoric acid and the alkylamide are byproducts of the methylation reagent, the triazine could be a soil degradation product of simazine, used in agricultural practices. A simazine concentration of $0.27 \mathrm{mg} / \mathrm{kg}$ soil was estimated (Trubetskaya and Trubetskov, personal communication). Pyrolysis/methylation drastically changed the pattern of pyrolysis products when compared with conventional pyrolysis, giving a cleaner chromatogram probably due to a lesser structural fragmentation. Alkanes, alkenes, and alkylbenzenes appeared as trace compounds. These results are coincident with those of Hatcher and Clifford (32) in spite of the use of a different pyrolysis system. The relative abundances of products in pyrolysis/methylation of the humic acid is about fifteen times higher than in conven- tional pyrolysis, which conclusively indicates that the distribution, composition, and amount of products are completely biased in the latter technique and would not be representative of the original composition of the humic acid. Hatcher and Clifford (32) confirmed that pyrolysis/ methylation produces $2-4$ times the product yields of conventional pyrolysis and arrived at a similar conclusion as ours.

The alkylated benzene and naphthalene series require separate mention. Although present, they do not contribute significantly to the bulk of the pyrolysate in any of the humic acids studied $(23,32)$, particularly in the methylated samples where the relative abundance of alkylbenzenes is 20 times lower than in conventional pyrolysis. It was proved that pyrolysis/methylation prevents the formation of alkylbenzenes through protection of carboxylic groups in model compounds (18). Therefore, identification of alkylbenzenes as the major series of compounds from the pyrolysis of fatty acids (18, 19) would indicate that these compounds are likely artifacts originated in the analytical procedure and would have no significance regarding structural studies.

From preliminary studies on pyrolysis/methylation of humic substances $(32,35,39,44)$, it can be concluded that the method reveals the presence in aliphatic and aromatic structures of carboxylic groups, which are usually absent from conventional pyrolysis. Also, the method does not completely protect carboxyl groups, since decarboxylation reactions were observed in model phenolic acids (39). Partial methylation of free phenolic hydroxyls was obtained, which is in accordance with previous reports (34, 35). The reason for the presence of unmethylated hydroxyls (which was also observed in MWL lignins, unpublished data) is unknown, as pyrolysis, even with an excess of TMAH, also yields phenols with free hydroxyls. Pyrolytic methylation of simple phenolic acid (e.g., vanillic acid) also resulted in derivatives with free hydroxyls. Therefore, the method has some limitations.

Finally, the finding of benzenecarboxylic acids through pyrolysis/methylation is in accordance with previous nondegradative fractionation (28), NMR (29) and oxidative methods (27), which validate both pyrolysis/methylation and to some extent chemical degradations. This was also supported by the data of Hatcher et al. (45) with a volcanic soil humic acid. In fact, the NMR spectrum clearly indicated that this humic acid is composed of only aromatic and carboxyl carbons. Permanganate oxidation indicated a predominantly aromatic character (29). Pyrolysis/ methylation yielded mainly benzenecarboxylic acid methyl esters, in accordance with both NMR and oxidation data.

\section{Significance of Pyrolysis/Methylation Products}

It seems appropriate to comment briefly on the significance of the finding of benzenecarboxylic acids in light of the structural scheme proposed by Schnitzer and Khan (1). The question arises as to whether benzenecarboxylic acids are present in the humic matrix in ester linkages and released upon hydrolysis [a reaction suggested by de Leeuw and Baas (35) for polyesters] or free and released by thermal desorption once the carboxylic groups have been protected. In this respect, it is worth mentioning that Schnitzer and Neyroud (21) stated that fatty acids are esterified to hydroxyl groups of phenolic acids. One could hypothesize that the identification of substantial 
amounts of aliphatic carboxylic, benzenecarboxylic, and phenolic acids in fulvic and humic acids supports this inference. Alternatively, in the case of a hydrogen-bonded structure, methylation and thermal evaporation of released benzenecarboxylic acids will yield similar products. Therefore, at this stage, pyrolytic methylation gives no conclusive information, if any, on the type of linkage of benzenecarboxylic acids.

In the past, benzenecarboxylic and aliphatic dicarboxylic acids recovered after oxidative degradations were considered to be the result of oxidative reactions on aromatic nuclei highly substituted by cross-linking aliphatic side chains, and the benzenecarboxylic acids were considered as useful guides to the chemical structure of the original humic acids (1). Subsequently, Schnitzer (27) hypothesized that benzenecarboxylic and phenolic acids recovered as degradation products of fulvic and humic acids originated from more complex aromatic structures or could have occurred in the initial humic materials in essentially the same forms in which they were isolated. The isolation of such compounds by nondegradative methods (28) and the data reported herein support the latter statement.

Pyrolysis/methylation corroborates the presence in the humic fractions of aromatic and aliphatic acids, which become apparent in pyrolysis only after protection of carboxylic and phenolic groups. These compounds are abundant in the chromatograms and would represent pristine components.

By comparing the data obtained by pyrolysis/methylation vs conventional pyrolysis, it is demonstrated that pyrolysis, as traditionally performed, is biased not only by the thermal degradation of functional groups mainly in benzenecarboxylic and phenolic acids that represent an important part of the macromolecule but also by the production of artifacts from fatty acids, thus concealing useful information on the chemical nature of humic substances and the biodegradation processes of organic matter leading to building blocks.

\section{Concluding Comments}

Finding new data by analytical pyrolysis of humic substances could mistakenly lead to the conclusion that the technique is able to solve structural uncertainties or be the panacea for structural studies. Although pyrolysis/ methylation has considerable potential for determining the chemical nature of relatively simple polymers (e.g., polymaleic acid, polyesters, etc.) or alternatively being used as an evaporation method for analysis of nonpolar compounds present in inorganic matrices, its application to complex macromolecules should be undertaken with care. This technique is a double-edged sword, as it can lead to errors if not accompanied by cautious study of the thermal behavior of the structural units.

One example of the potentialities of pyrolysis is the recent finding of phenolic and benzenecarboxylic acids in the pyrolytic methylation of aquatic and terrestrial humic substances. The use of pyrolysis together with derivatization methods, high-temperature GC columns, etc., indicates that there is still a lot to be explored before the possibilities of analytical pyrolysis in the study of humic substances run out.

Pyrolysis/methylation is an easy derivatization method which provides complementary information to that obtained by conventional pyrolysis. It is suggested that previous data obtained by conventional pyrolysis studies and statements or structural models formulated on them are incomplete and must be revised to conform with the new insights offered by pyrolysis/methylation. The identification of benzenecarboxylic acids in different fulvic and humic samples is of interest as it validates model studies regarding interaction of carboxyl and hydroxyl groups in aromatic units with metals, minerals, pollutants, and biocides.

\section{Acknowledgments}

The author gratefully acknowledges Dr. O. E. Trubetskaya and 0 . A. Trubetskoj, Moscow, for the use of pyrolysis data from the agricultural soil humic acid obtained in a joint research.

\section{Literature Cited}

(1) Schnitzer, M.; Khan, S. U. Humic Substances in the Environment; Marcel Dekker: New York, 1972.

(2) Norwood, D. L. In Humic Substances and their Role in the Environment; Frimmel, F. H., Christman, R. F., Eds.; Wiley: Chichester, 1988; pp 133-148.

(3) Martin, F.; Saiz-Jimenez, C.; Cert, A. Soil Sci. Soc. Am. J. 1979, 43, 309-312.

(4) Saiz-Jimenez, C.; de Leeuw, J. W. J. Anal. Appl. Pyrolysis 1986, 9, 99-119.

(5) Schulten, H. R.; Plage, B.; Schnitzer, M. Naturwissenschaften 1991, 78, 311-312.

(6) Irwin, W. J. J. Anal. Appl. Pyrolysis 1979, 1, 3-25.

(7) Saiz-Jimenez, C.; de Leeuw, J. W. Org. Geochem. 1984, 6, 287-293.

(8) Saiz-Jimenez, C.; de Leeuw, J. W. J. Anal. Appl. Pyrolysis 1987, 11, 367-376.

(9) Tegelaar, E. W.; de Leeuw, J. W.; Saiz-Jimenez, C. Sci. Total Environ. 1989, 81/82, 1-17.

(10) Pouwels, A. D.; Eijkel, G. B.; Boon, J. J. J. Anal. Appl. Pyrolysis 1989, 14, 237-280.

(11) Boon, J. J.; de Leeuw, J. J. J. Anal. Appl. Pyrolysis 1987, $11,313-327$.

(12) Saiz-Jimenez, C. In Humus, its Structure and Role in Agriculture and Environment; Kubát, J., Ed.; Elsevier: Amsterdam, 1992; pp 27-38.

(13) De Leeuw, J. W.; van Bergen, P. F.; van Aarssen, B. G. K.; Gatellier, J. P. L.A.; Sinninghe-Damste, J. S.; Collinson, M. E. Philos. Trans. R. Soc. London B 1991, 333, 329-337.

(14) Boon, J. J. In Microbial Mats: Stromatolites; Cohen, Y., Castenholz, R. W., Halvorson, H. O., Eds.; Alan R. Liss: New York, 1984; pp 313-342.

(15) Chiavari, G.; Galleti, G. C. J. Anal. Appl. Pyrolysis 1992, $24,123-137$.

(16) Saiz-Jimenez, C.; de Leeuw, J. W. Org. Geochem. 1984, 6, $417-422$.

(17) Saiz-Jimenez, C.; de Leeuw, J. W. Org. Geochem. 1986, 10, 869-876.

(18) Saiz-Jimenez, C. Org. Geochem., in press.

(19) Saiz-Jimenez, C. Naturwissenschaften, in press.

(20) Grimalt, J. O.; Saiz-Jimenez, C. Sci. Total Environ. 1989, $81 / 82,409-420$.

(21) Schnitzer, M.; Neyroud, J. A. Fuel 1975, 54, 17-19.

(22) Grimalt, J. O.; Hermosin, B.; Yruela, I.; Saiz-Jimenez, C. Sci. Total Environ. 1989, 81/82, 421-428.

(23) Saiz-Jimenez, C. In Humic Substances in the Global Environment and Implications on Human Health; Senesi, N., Miano, T. M., Eds.; Elsevier: Amsterdam, 1994; pp 7190 .

(24) Hawthorne, S. B.; Miller, D. J.; Barkley, R. M.; Krieger, M. S. Environ. Sci. Technol. 1988, 22, 1191-1196.

(25) Schlautman M. A.; Morgan, J. J. Environ. Sci. Technol. 1993, 27, 961-969.

(26) De Leeuw, J. W.; de Leer, E. W. B.; Sinninghe-Damsté, J. S.; Schuyl, P. J. W. Anal. Chem. 1986, 58, 1852-1857. 
(27) Schnitzer, M. In Soil Organic Matter; Schnitzer M., Khan, S. U., Eds.; Elsevier: Amsterdam, 1978; pp 1-64.

(28) Ogner, G.; Schnitzer, M. Can. J. Chem. 1971, 49, 10531063.

(29) Hatcher, P. G.; Schnitzer, M.; Dennis, L. W.; Maciel, G. E. Soil Sci. Soc. Am. J. 1981, 45, 1089-1094.

(30) Saiz-Jimenez, C.; de Leeuw, J. W. Sci. Total Environ. 1987, $62,115-119$

(31) Saiz-Jimenez, C. Atmos. Environ. 1993, 27B, 75-85.

(32) Hatcher P. G.; Clifford, D. J. Org. Geochem., in press.

(33) Challinor, J. M. J. Anal. Appl. Pyrolysis 1989, 16, 323-333.

(34) Mulder, M. M.; van der Hage, E. R. E.; Boon, J. J. Phytochem. Anal. 1992, 3, 165-172.

(35) De Leeuw, J. W.; Baas, M. J. Anal. Appl. Pyrolysis 1993, 26, 175-184.

(36) Anderson, K. B.; Winans, R. E. Anal. Chem. 1991, 63, 29012908.

(37) Ohtani, H.; Fujii, R.; Tsuge, S. J. High Resolut. Chromatogr. 1991, 14, 388-391.

(38) Saiz-Jimenez, C.; Hermosin, B.; Ortega-Calvo, J. J. Water Res. 1993, 27, 1693-1696.
(39) Saiz-Jimenez, C. Environ. Sci. Technol. 1994, 28, 97-100.

(40) Braun, D.; Pomakis, J. Makromol. Chem. 1974, 175, 14111425.

(41) Anderson, H. A.; Russell, J. D. Nature 1976, 260, 597.

(42) Bracewell, J. M.; Robertson G. W.; Welch, D. I. J. Anal. Appl. Pyrolysis 1980, 2, 239-248.

(43) Trubetskoj, O. A.; Trubetskaya, O. E.; Khomutova, T. E. Soil Biol. Biochem. 1992, 24, 893-896.

(44) Saiz-Jimenez, C.; Ortega-Calvo, J. J.; Hermosin, B. Naturwissenschaften 1994, 81, 28-29.

(45) Hatcher, P. G.; Faulon, J. L.; Clifford, D. A.; Mathews, J. P. In Humic Substances in the Global Environment and Implications on Human Health; Senesi, N., Miano, T.M., Eds.; Elsevier: Amsterdam, 1994; pp 133-138.

Received for review January 14, 1994. Accepted June 28, 1994.

Abstract published in Advance ACS Abstracts, August 1, 1994. 